
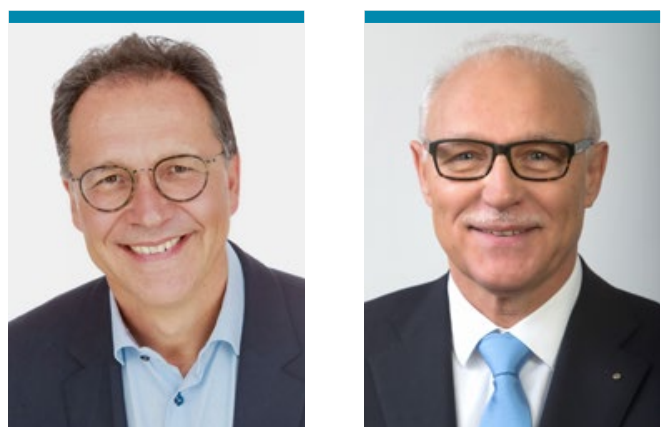

„Treffen Sie in Wiesbaden alte und neue Freunde, die mit Ihnen das Interesse an der Allergologie teilen und lassen Sie sich von der Allergologie neu begeistern!"

Prof. Dr. Ludger Klimek, Zentrum für Rhinologie und Allergologie Wiesbaden, Kongresspräsident

Prof. Dr. Wolfgang Wehrmann, Dermatologische Gemeinschaftspraxis Münster,

Kongresspräsident

\title{
Gemeinsam die Allergologie stärken!
}

$\mathrm{H}$ (AeDA), der Deutschen Gesellschaft für Allergoloweltmedizin (GPA). Ihre Teilnahme an der wichtigsten allergologischen Fortbildungsveranstaltung in Deutschland entspricht unserem Motto: Gemeindung - Versorgung. zeigt die Bedeutung unseres interdisziplinären Querschnittsfaches. Hier werden Ihnen die hohe allergologischen Laboren dieser Welt nutzbar gemacht für Ihre praktische Fortbildung und die Verne Kosten kommen: Egal ob Sie Neueinsteiger oder

\section{Inhaltsverzeichnis}

Thema

Allergologie - Kasuistiken

Spezifische Immuntherapie mit Allergenen

Allergie und Umwelt

Allergie - Diagnostik

Immunologie

Allergologie - Therapie

Verzeichnis der Autoren erzlich Willkommen zum 12. Deutschen Allergiekongress in Wiesbaden - im Namen des Ärzteverbands Deutscher Allergologen gie und klinische Immunologie (DGAKI) und der Gesellschaft für Pädiatrische Allergologie und Umsam die Allergologie stärken! Forschung - Fortbil-

Ihr Kongressbesuch stärkt die Allergologie und Wissenschaft und neueste Erkenntnisse aus den sorgung unserer Patienten. Jeder wird hier auf sei-
Postennummer

P01-P12

P13-P26, P75, P76, P79, P87

P27-P37, P74, P83

P38-P49, P73, P85, P86

P50-P59, P72, P80

P60-P70, P77, P78, P81, P82

\section{Seite}

55

59

67

72

79

83

90 „alter Hase“ in der Allergologie sind, Sie erhalten viel Raum für Fragen, Kommentare und Diskussionen im interaktiven und interdisziplinären Austausch.

Der Deutsche Allergiekongress findet auf höchstem Niveau statt und bietet eine Übersicht über die aktuellen wissenschaftlichen Entwicklungen in Verbindung mit praktischer Fortbildung und zahlreichen Workshops. Die Abstracts vom Allergiekongress finden Sie ab nebenstehender Seite 55.

Der interdisziplinäre Charakter der Allergologie ermöglicht den Blick über den Tellerrand: Sie werden nicht nur aus Ihrem eigenen Fachgebiet, sondern auch von den allergologischen Nachbarfächern spannende Neuigkeiten erfahren und praktische Tipps bekommen. Wo sonst haben Sie die Gelegenheit, über die Grenzen des eigenen Fachs hinauszuschauen und praktische Erfahrungen auch außerhalb der eigenen Fachdisziplin zu sammeln?

Verpassen Sie am Freitag nach einem spannenden Kongresstag keinesfalls den Gesellschaftsabend in der „Villa im Tal“! Bei Livemusik und exzellentem Catering darf gefeiert werden.

Sie finden in Wiesbaden ideale Bedingungen zum wissenschaftlichen und fachlichen Networking: Treffen Sie in Wiesbaden alte und neue Freunde, die mit Ihnen das Interesse an der Allergologie teilen und lassen Sie sich von der Allergologie neu begeistern! Erleben Sie einen Kongress inmitten von historischen Gebäuden in Jugendstil, Barock und Klassizismus in der Altstadt von Wiesbaden!
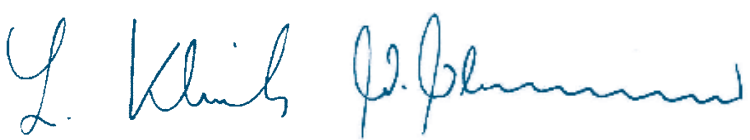